\title{
Dental infections in children could indicate neglect issues
}

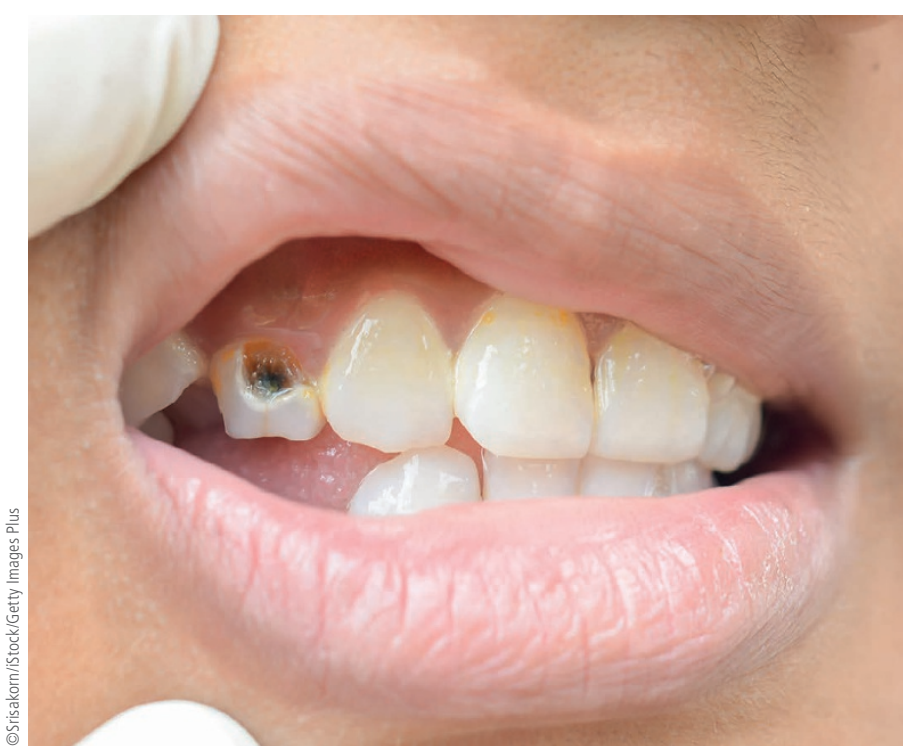

Dental infections in children could be a sign of potential neglect and should be referred to safeguarding professionals, suggests original research published in this issue of the BDJ.

Researchers from King's College London found that a significant number of children admitted for maxillofacial space infection were already known to social services.

Child neglect has a significant impact on children's physical and emotional health and development with lifelong consequences. In March 2017, 51,080 children were subject to a child protection plan in England and in $48.1 \%$ of these cases, neglect was the initial category of abuse.

Neglect is defined as the persistent failure to meet a child's basic physical and/or psychological needs, likely to result in the serious impairment of the child's health or development. Neglect also includes not providing access to healthcare.

Similarly, dental neglect is defined as the persistent failure to meet a child's basic oral health needs, likely to result in the serious impairment of the child's oral or general health or development. Dental decay can lead to maxillofacial space infections - a potential sign of dental neglect.

Researchers from King's College Hospital's London's Department of Oral and Maxillofacial Surgery, Guy's and St Thomas NHS Trust, King's College Dental Institute, and King's College London's School of Tissue Engineering \& Biophotonics Dental Institute carried out a retrospective observational study.

They reviewed the records of all children under the age of 16, who were admitted as an emergency under oral and maxillofacial surgery at King's College Hospital between January 2015 and January 2017 and required incision and drainage of a dental/maxillofacial space infection under general anaesthesia, of odontogenic cause.

The researchers set out to assess what proportion of these children were already known to social services for neglect and to support the development of a local safeguarding referral protocol for children with dental neglect admitted under oral and maxillofacial surgery.

For the study, 27 children were included and 11 children (40\%) were known to social services.
Results showed that five patients out of the 27 were discussed with a Trust safeguarding team member during their hospital stay and of these five patients, one new referral to social services was made and three cases were re-referred due to new safeguarding concerns.

On average, 3.2 teeth were extracted with an average hospital stay of 2.5 days.

The researchers said: 'Our study found, that $40 \%$ of children who presented with potential life-threatening dental/maxillofacial space infections were already known to social services. More than 50\% of these children were between five and eight years old, suggesting this group are at greater risk of harm and highlighting a potential coincidence of dental neglect and broader global neglect.

'Where parents or carers repeatedly fail to access dental treatment for a child's tooth decay or leave dental tooth pain untreated, 'alarm bells' should ring for clinicians to consider neglect.'

They concluded: 'Our recommendation is that all children admitted with dental/maxillofacial space infections, where dental neglect may be present, should be discussed with the local safeguarding team.

'Where there is evidence of dental neglect or broader signs of neglect, referral to the safeguarding team should be made.'

1. Schlabe J, Kabban M, Chapireau D, Fan K. Paediatric dental infections - a potential too for identifying children at risk of neglect? Br Dent J 2018; 225: 757-761. Available at https://www.nature.com/articles/sj.bdj.2018.862. 